\title{
Sanitary state of pig herds from Brittany. 1. Lesions of the respiratory tract in fattening pigs
}

\author{
F. MADEC (1), P. ROBINEAU ${ }^{(2)}$, A. QUERREC ${ }^{(2)}$, J.F. PANSART ${ }^{(1)}$
}

(1) Station de Pathologie Porcine, B.P. 9, 22440 Ploufragan

(2) Direction des Services Vétérinaires des Côtes-du-Nord, 8, Place du 71-R.I., 22021 Saint-Brieuc

\begin{abstract}
Examination of the respiratory tract of slaughter pigs was performed in six slaughter-houses of Brittany (France) during April 1987. A total of 249 batches of pigs representative of the slaughter population of this region was selected. In each batch, 25 lungs were randomly selected and 10 snouts. The latter were sawn for analysis. Pneumonia lesions were recorded lobe by lobe according to the extent of consolidation from 0 (no lesion) to 4 (all the lobe consolidated). Rhinitis was alloted a score of 0 to 4 according to the degree of atrophy of the 4 turbinates and from 0 to 2 according to the dystrophy of the septum.

A prevalence of 48.1 p. 100 was obtained for pneumonia ( $n=6014$ pigs examined). Pleuritis was noted in 14 p. 100 of the pigs and atrophic rhinitis in 44 p. 100 of them. Some associations were found and discussed. Thus, a higher incidence of pneumonia was recorded in the batches of pigs the most affected by rhinitis. Pigs coming from areas with a high density were also more concerned as well as those coming from breeding-finishing herds.
\end{abstract}

\section{Antigenic characterization of porcine respiratory coronavirus using monoclonal antibodies directed against TGEV}

\author{
H. LAUDE, Jacqueline GELFI, D. RASSCHAERT, B. DELMAS \\ Institut National de la Recherche Agronomique. Station de Recherches de Virologie \\ et d'Immunologie, route de Thiverval, 78850 Thiverval-Grignon
}

PRCV, a recently identified porcine respiratory coronavirus, has become widespread in European pig producing countries. The infection results in the induction of antibodies neutralizing the enteric coronavirus TGEV (Transmissible Gastroenteritis Coronavirus). The antigenic relationship between PRCV and TGEV was investigated in an indirect immunofluorescence assay using a panel of monoclonal antibodies raised against the Purdue strain of TGEV. The three isolates of PRCV exhibited an identical antigenic spectrum. Several differences with TGEV strains were noted, the most relevant of which affects the major antigenic site D of the spike glycoprotein E2. Several hypotheses about the possible derivative source of this virus are discussed.

\section{Effect of health problems on the growth of pigs between weaning and slaughter}

\author{
P. LE FOLL, N. AMARA, B. GIRAL, T. SOLIGNAC
}

Institut Technique du Porc, 34, boulevard de la Gare, 31500 Toulouse

An epidemiological survey of breeding-finishing pig herds was conducted in 1986 in the MidiPyrenees region. The survey concerned 550 animals studied individually from birth to slaughter and belonging to 46 herds. The purpose was to determine the influence of health problems on growth performance. 